\title{
Molecular-beam epitaxial growth and characteristics of highly uniform InAs/GaAs quantum dot layers
}

\author{
Zetian Mi and Pallab Bhattacharya ${ }^{\text {a) }}$ \\ Department of Electrical Engineering and Computer Science, The University of Michigan, Ann Arbor, \\ Michigan 48109-2122
}

(Received 22 December 2004; accepted 1 June 2005; published online 21 July 2005)

\begin{abstract}
We have investigated the molecular-beam epitaxial growth and structural and photoluminescence characteristics of self-organized InAs/GaAs quantum dot bilayers, in which a first seed layer of stressor dots is followed by a GaAs barrier layer and a second layer of active dots. At room temperature, the peak emission wavelength is at $\sim 1.4 \mu \mathrm{m}$. By optimizing the growth parameters for both dot layers and the GaAs barrier layer, we have measured photoluminescence linewidths of 10.6 and $17.5 \mathrm{meV}$ at 20 and $300 \mathrm{~K}$, respectively. The measurement and analysis of temperature-dependent photoluminescence data indicate that there is no observable carrier redistribution amongst the dots with an increase of temperature and there is a high degree of size uniformity. The photoluminescence linewidth of $17.5 \mathrm{meV}$ at $300 \mathrm{~K}$ is almost identical to that measured in the emission from a single dot, indicating that the linewidth is determined by homogeneous broadening. (C) 2005 American Institute of Physics. [DOI: 10.1063/1.1985969]
\end{abstract}

\section{INTRODUCTION}

Self-organized quantum dots, formed in the StranskiKrastanow growth mode during epitaxy of strained $\operatorname{In}(\mathrm{Ga}) \mathrm{As} / \mathrm{GaAs}$ heterostructures, are used as the active region of lasers, ${ }^{1-3}$ amplifiers, ${ }^{4-6}$ infrared detectors, ${ }^{7-9}$ and in other device applications. ${ }^{10-14}$ The size and shape of the coherently strained islands, which enable three-dimensional quantum confinement, depend on the heterostructures and the conditions for epitaxy. Island formation is kinetically controlled, based on arguments of minimization of crystal free energy by the growth front. There is therefore a distribution in the size of the islands in a given layer and also a randomness in their spatial ordering. The size distribution gives rise to an inhomogeneous broadening of optical transitions resulting from carrier recombination or generation. Linewidths (full width at half maximum, FWHM) in the range of 30-60 meV are commonly measured in the photoluminescence spectra of $\operatorname{In}(\mathrm{Ga}) \mathrm{As} / \mathrm{GaAs}$ quantum dots. ${ }^{15,16}$ The gain of a laser is inversely proportional to the linewidth of the spontaneous emission spectrum. Similarly, the intersubband absorption coefficient, which becomes relevant in intersubband quantum dot infrared detectors, is also inversely proportional to the transition linewidth. Therefore, it is imperative that techniques to reduce the transition linewidths are explored. In this regard, considerable success has been reported recently. ${ }^{17-23}$ In the present study, we have examined the growth of quantum dot heterostructures, with the objective of achieving uniformity in the dot size, and have made detailed temperature-dependent photoluminescence measurements of the quantum dot heterostructures. We measure a photoluminescence linewidth of the dot ensemble of $10.6 \mathrm{meV}$ at $20 \mathrm{~K}$, which is limited by inhomogeneous

${ }^{a)}$ Electronic mail: pkb@eecs.umich.edu broadening, and a linewidth of $17.5 \mathrm{meV}$ at $300 \mathrm{~K}$, which is very close to the homogeneous linewidth for emission from a single quantum dot.

\section{EPITAXIAL GROWTH OF InAs/GaAs QUANTUM DOT BILAYERS}

In the quest for achieving uniformity in the dot size and thereby reducing the broadening of the photoluminescence spectrum of an ensemble of quantum dots, techniques such as vertical coupling of dot layers ${ }^{24-26}$ and the use of buried stressor dot layers ${ }^{17-19}$ have been explored. These techniques of "strain patterning" rely on the fact that the strain field around the quantum dots in one layer alters the adatom migration rates of subsequent dot layers such that larger and more uniform dots are formed on these layers. However, complete elimination of the randomness of dot formation will probably be impossible since thermal diffusion effects will be present. The best results, in terms of dot uniformity, have been obtained with a quantum dot bilayer system, ${ }^{17,19}$ in which, the "stressor dots" in the first layer act as a template which influences the growth kinetics, formation, and characteristics of the second layer of "active dots." For the growth of $\operatorname{In}(\mathrm{Ga})$ As/GaAs quantum dots, there is a tensile strain field in the GaAs barrier above the dots in the first layer, which influences adatom migration and induces a vertical coupling of the second layer of dots. By tuning the growth parameters used to form the first layer of stressor dots, which influence their size, shape, and density, and by carefully adjusting the thickness of the GaAs barrier layer in between, the size nonuniformity of the second layer of active dots can be minimized. For example, with a higher growth temperature and lower growth rate, it is expected that the stressor dots will be wider in lateral extent and will produce a wider strain field in the GaAs cap layer. The thickness of the latter also determines the tensile strain field that promotes vertical coupling of the active dots. The extent of strain-free 


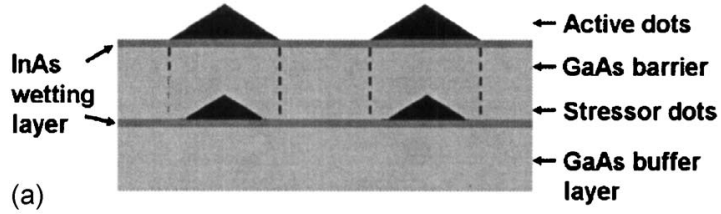

(b)

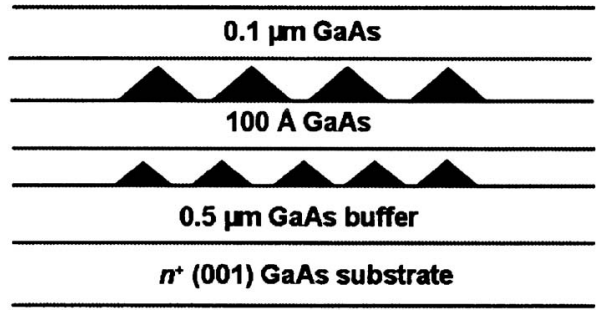

FIG. 1. (a) Schematic of a bilayer quantum dot heterostructure that shows perfect vertical coupling of the active (second) quantum dot layer due to the strain field generated by the stressor quantum dots. The dashed lines indicate the lateral extent of the tensile strain field in the GaAs barrier; (b) quantum dot heterostructure grown by molecular-beam epitaxy.

regions in the barrier layer, in between the dots, should be minimized, so that the nucleation and formation of dots with random sizes in these regions are suppressed. The dot bilayer heterostructure is illustrated in Fig. 1(a).

The quantum dot heterostructures investigated in the present study consist of two layers of InAs/GaAs quantum dots (QDs) separated by a $100-\AA$ GaAs spacer layer, as shown in Fig. 1(b). These structures were grown on (001) $n^{+}$-GaAs substrates in a Veeco Modular Gen II molecularbeam epitaxy (MBE) system with an $\mathrm{As}_{2}$ overpressure of 8 $\times 10^{-6}$ Torr. Following the growth of a $0.5-\mu \mathrm{m}$ GaAs buffer layer at $600{ }^{\circ} \mathrm{C}$, the substrate temperature was lowered for the growth of the first (seed or stressor) InAs QD layer. This layer was grown at different temperature $(500,520$, and $\left.535^{\circ} \mathrm{C}\right)$ with different InAs growth rates $(0.02,0.015$, and 0.01 monolayer $(\mathrm{ML}) / \mathrm{s}$ ) for samples $\mathrm{A}, \mathrm{B}$, and $\mathrm{C}$, respectively. After the deposition of 2.4 MLs of InAs, a $100-\AA$ GaAs cap layer was grown. The substrate temperature was raised and the sample was annealed at $600{ }^{\circ} \mathrm{C}$ for $10 \mathrm{~min}$. This annealing procedure was found to be critical in removing surface undulations and defects induced in the GaAs cap by the first QD layer. ${ }^{19}$ The substrate temperature was then lowered to $480{ }^{\circ} \mathrm{C}$ and 3.1 MLs of InAs were deposited to form the second (or active) QD layer with the same growth rate as that for the first QD layer. The second QD layer was capped with $200-\AA$ GaAs at the lower growth temperature before the substrate temperature was raised to $600{ }^{\circ} \mathrm{C}$ again for the growth of an additional $0.1-\mu \mathrm{m}$ GaAs layer. The sample description and growth parameters are listed in Table I.

\section{STRUCTURAL CHARACTERIZATION}

We will first describe and discuss the structural characteristics of the stressor and active quantum dots in the different samples. Figures 2(a)-2(c) show the surface atomic force microscopy (AFM) images of the stressor dot layers in samples A, B, and C, respectively. In these control samples, only the first layer of stressor dots was grown for the AFM measurements. Careful examination of the data reveals that
TABLE I. Growth parameters for the first (stressor) and second (active) quantum dot layers of samples $\mathrm{A}, \mathrm{B}$, and $\mathrm{C}$.

\begin{tabular}{ccccccc}
\hline \hline & $\begin{array}{c}\text { Growth } \\
\text { rate }\end{array}$ & \multicolumn{2}{c}{$\begin{array}{c}\text { Stressor } \\
\text { QD }\end{array}$} & \multicolumn{2}{c}{$\begin{array}{c}\text { Active } \\
\text { QD }\end{array}$} & $\begin{array}{c}\text { FWHM } \\
(20 \mathrm{~K})\end{array}$ \\
\hline & $(\mathrm{ML} / \mathrm{s})$ & $\begin{array}{c}T_{g} \\
\left({ }^{\circ} \mathrm{C}\right)\end{array}$ & $\mathrm{ML}$ & $\begin{array}{c}T_{g} \\
\left({ }^{\circ} \mathrm{C}\right)\end{array}$ & $\mathrm{ML}$ & $(\mathrm{meV})$ \\
& & 500 & 2.4 & 480 & 3.1 & 20 \\
$\mathrm{~A}$ & 0.02 & 520 & 2.4 & 480 & 3.1 & 14 \\
$\mathrm{~B}$ & 0.015 & 535 & 2.4 & 480 & 3.1 & 10.6 \\
$\mathrm{C}$ & 0.01 & 535 & & & &
\end{tabular}

with an increase of growth temperature and reduction of growth rate, the size and size uniformity of the dots increase and their density decreases. The average widths are approximately $22,24.5$, and $28 \mathrm{~nm}$; average heights are 6.1, 7.0, and $8.0 \mathrm{~nm}$; and average densities are $2.6 \times 10^{10}, 1.7 \times 10^{10}$, and $1.0 \times 10^{10} \mathrm{~cm}^{-2}$, for control samples of the stressor dot layers in samples A, B, and C, respectively. Figure 2(d) shows the surface AFM image of the second layer of active dots of sample C. It is evident that the dots are large and very uniform, with an average height of $12.5 \mathrm{~nm}$, width of $43 \mathrm{~nm}$, and density of $1.0 \times 10^{10} \mathrm{~cm}^{-2}$. With a higher growth temperature and lower growth rate, the larger stressor dots produce a larger strain field in the GaAs barrier layer. This promotes increased stress-directed In migration towards regions

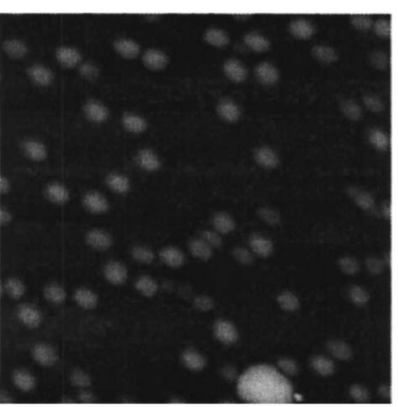

$0.5 \mu \mathrm{m} \times 0.5 \mu \mathrm{m}$

(a)

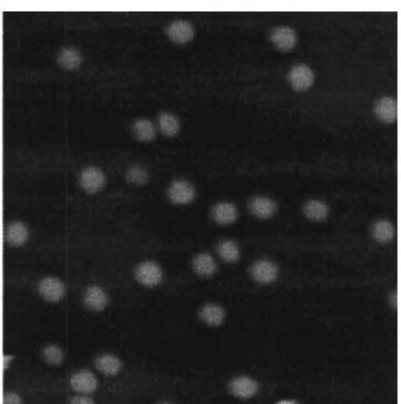

$0.5 \mu \mathrm{m} \times 0.5 \mu \mathrm{m}$

(c)

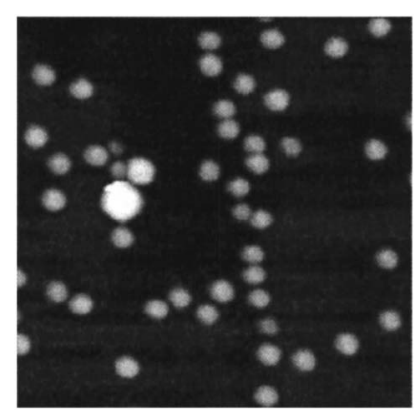

$0.5 \mu \mathrm{m} \times 0.5 \mu \mathrm{m}$

(b)

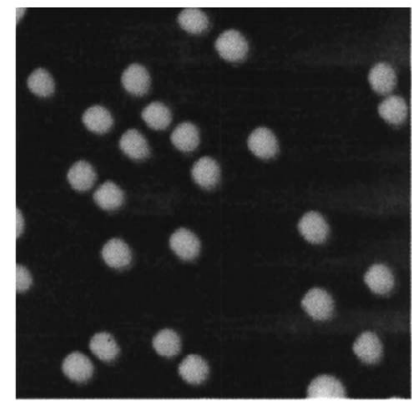

$0.5 \mu \mathrm{m} \times 0.5 \mu \mathrm{m}$

(d)
FIG. 2. AFM images of the stressor quantum dot layers of (a) sample A, (b) sample B, (c) sample C, and (d) the active quantum dot layer of sample C. These AFM samples were grown under identical conditions to those for the photoluminescence experiments except that no GaAs cap layer was grown. The scan field is $0.5 \times 0.5 \mu \mathrm{m}^{2}$. The average widths are approximately 22 , 24.5 , and $28 \mathrm{~nm}$; average heights are $6.1,7.0$, and $8.0 \mathrm{~nm}$; and average densities are $2.6 \times 10^{10}, 1.7 \times 10^{10}$, and $1.0 \times 10^{10} \mathrm{~cm}^{-2}$, for the stressor dot layers in samples $\mathrm{A}, \mathrm{B}$, and $\mathrm{C}$, respectively. The active dots of sample $\mathrm{C}$ have an average height of $12.5 \mathrm{~nm}$, width of $43 \mathrm{~nm}$, and density of 1.0 $\times 10^{10} \mathrm{~cm}^{-2}$. 


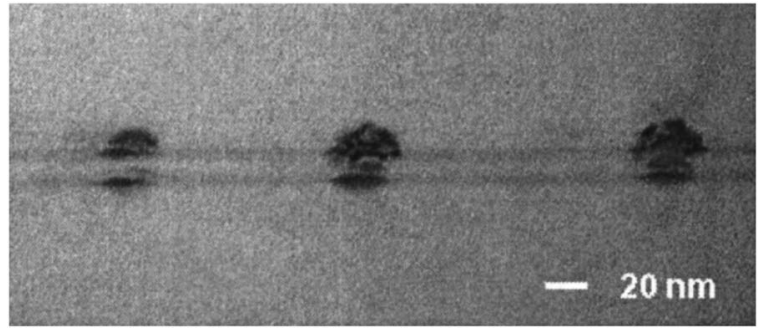

FIG. 3. Cross-sectional bright-field TEM image of sample C, showing the two coupled dot layers.

above the stressor dots, resulting in larger, taller, and more uniform active dots. The dots in the second layer are larger than the dots in the first seed layer also because for the same amount of adatom charge, the two-dimensional (2D) to island growth mode transition occurs earlier due to the strain field above the seed dots. Figure 3 shows a bright-field transmission electron microscopy (TEM) image of sample C. The perfect vertical coupling of the dots in the two layers and the increase in the size of the dots in the second layer are evident.

\section{TEMPERATURE-DEPENDENT PHOTOLUMINESCENCE: RESULTS AND DISCUSSION}

The photoluminescence properties of the QD heterostructures are next described. Photoluminescence was measured with a 1-m scanning spectrometer, an argon-ion $(\sim 480 \mathrm{~nm})$ excitation source, lock-in amplification, and detection with a liquid-nitrogen-cooled $\mathrm{Ge}$ detector. The samples were mounted on a cold finger in a variabletemperature closed-cycle liquid-He cryostat. The photoluminescence (PL) spectra measured at room temperature $(300 \mathrm{~K})$ and $20 \mathrm{~K}$ are shown in Figs. 4(a) and 4(b), respectively. It may be noted that the incident excitation intensities are $15 \mathrm{~W} / \mathrm{cm}^{2}$ and $1 \mathrm{~mW} / \mathrm{cm}^{2}$ at 300 and $20 \mathrm{~K}$, respectively. Several features in these spectra deserve attention. The two peaks, observed clearly in the room-temperature spectra of all the samples [Fig. 4(a)], arise from the transition involving the electron-hole ground state and the first excited states. The emission intensity is very strong at room temperature and it is noteworthy that the ground-state peak emission is at $1.4 \mu \mathrm{m}$. With careful tailoring of the growth parameters and heterostructure, the emission can be moved to longer wavelengths. The excited-state transitions are not observed in the low-temperature spectra of Fig. 4(b) since all the carriers are in the ground states and the excitation intensity is low. The peak intensities remain constant in all the three samples at room temperature, indicating a high degree of dot reproducibility in spite of the slightly altered growth conditions. It is observed that at both measurement temperatures, there is a systematic redshift of the PL peaks as one goes from sample A to $\mathrm{C}$. This redshift confirms the increasing dot size observed in the AFM images. Finally, the PL linewidths (FWHM) of the ground-state transitions are 10.6 and $17.5 \mathrm{meV}$ at 20 and $300 \mathrm{~K}$, respectively. These are the smallest linewidths measured in InAs or InGaAs self-organized
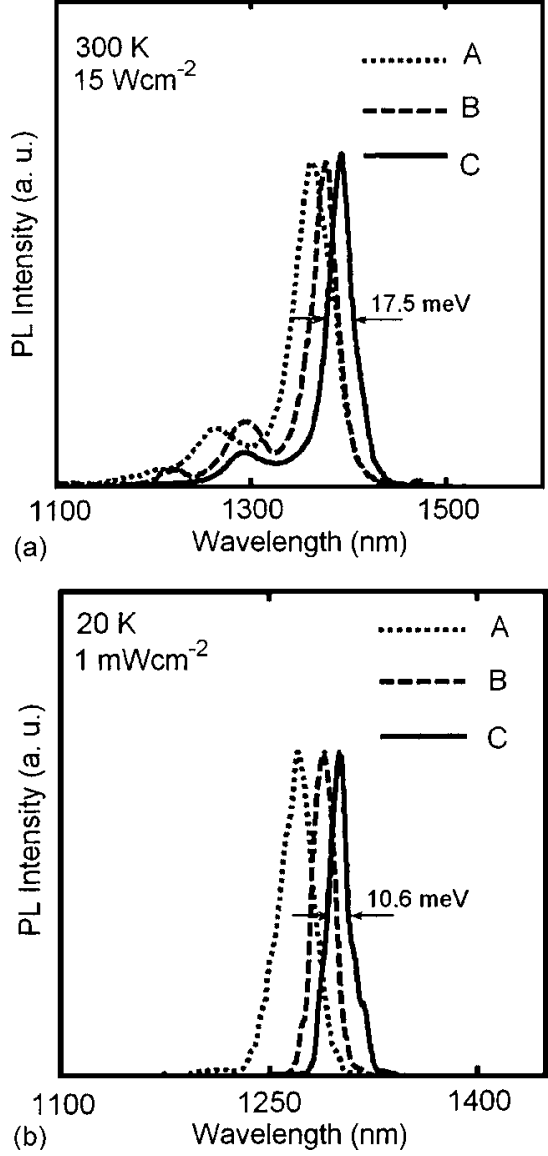

FIG. 4. Photoluminescence spectra of the samples measured at (a) 300 and (b) $20 \mathrm{~K}$. Sample $\mathrm{C}$ has the smallest linewidth at both $20 \mathrm{~K}(10.6 \mathrm{meV})$ and $300 \mathrm{~K}(17.5 \mathrm{meV})$. The incident excitation intensities are $1 \mathrm{~mW} / \mathrm{cm}^{2}$ at $20 \mathrm{~K}$ and $15 \mathrm{~W} / \mathrm{cm}^{2}$ at $300 \mathrm{~K}$

quantum dots, as compared to the linewidths of $14 \mathrm{meV}$ at $10 \mathrm{~K}$ reported by Le $\mathrm{Ru}$ et al. ${ }^{19}$ and $18.0 \mathrm{meV}$ at $300 \mathrm{~K}$ reported by Yang et al. ${ }^{20}$

Temperature-dependent PL data are shown in Figs. 5(a) and 5(b). The incident excitation intensity was held constant throughout the entire temperature range for these measurements. It is observed in Fig. 5(a) that the nature and rate of decay of the integrated PL intensity are identical for the ground- and first excited-state transitions. Thermal quenching of photoluminescence intensity is generally attributed to the presence of nonradiative defects and deep-level traps. A fast decay indicates nonradiative transitions associated with deep (nonhydrogenic) levels, while a slow decay with increasing temperature reflects the normal thermal quenching due to shallow levels. In addition, nonradiative channels in the GaAs barriers may contribute to the quenching. The quenching behavior in our sample is very complex and cannot be modeled simply in terms of an Arrhenius plot.

The temperature dependence of the photoluminescence emission linewidth is plotted in Fig. 5(b). The entire data were recorded with an incident excitation intensity of $50 \mathrm{~mW} / \mathrm{cm}^{2}$ and therefore the linewidth of $12.5 \mathrm{meV}$ at $20 \mathrm{~K}$ is larger than $10.6 \mathrm{meV}$ measured at the same temperature with an incident intensity of $1 \mathrm{~mW} / \mathrm{cm}^{2}$. There are several notable features in the data of Fig. 5(b). First, we do not observe the characteristic dip that is commonly observed in 

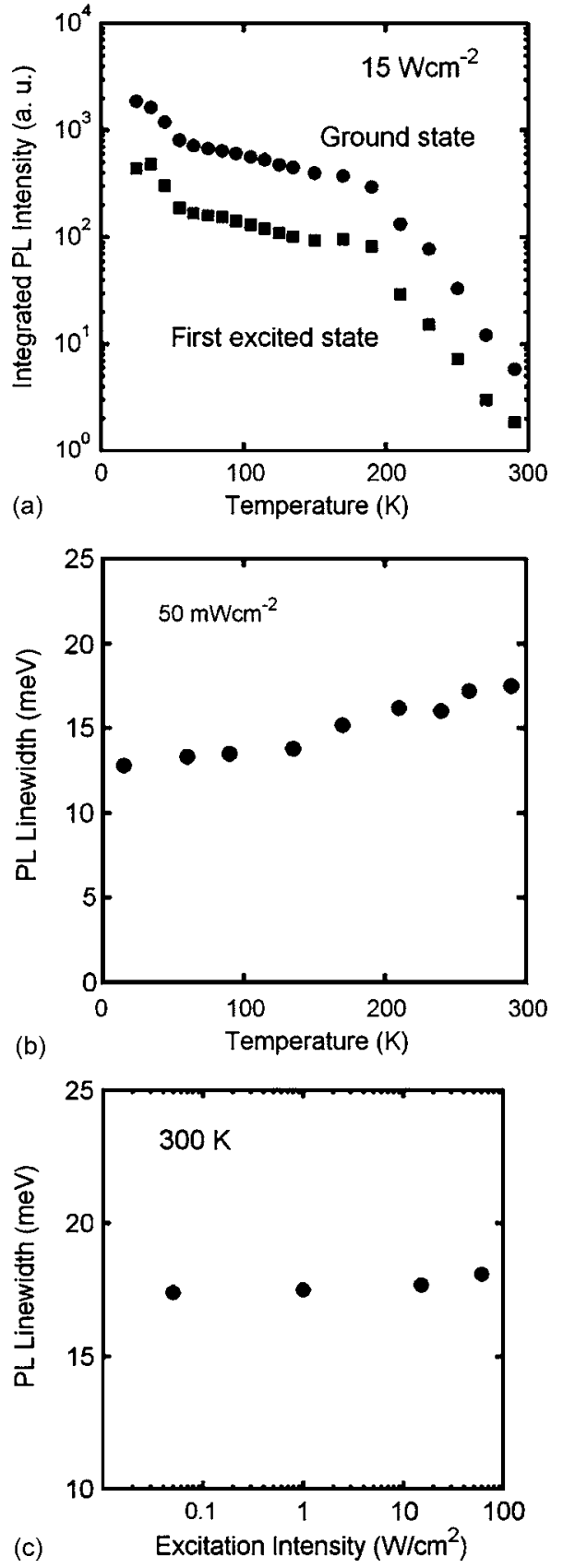

FIG. 5. Variation of (a) integrated photoluminescence intensity, (b) photoluminescence linewidth (FWHM) with temperature, and (c) photoluminescence linewidth with excitation intensity for sample C.

the temperature range of $100-200 \mathrm{~K}$, depending on the dot heterostructure. ${ }^{27,28}$ This dip is generally attributed to carrier redistribution among the dots with an increase of temperature. ${ }^{27,28}$ At very low temperatures, photoexcited carriers are frozen randomly into the dot states in a nonequilibrium distribution. With increasing temperature, some of these carriers are thermally activated out of the dots and into the wetting layer and GaAs barrier and are transported by hopping conduction predominantly to the larger dots with lower emission energy (higher binding energy). Therefore, a decrease of the PL linewidth is generally observed at $300 \mathrm{~K}$, compared to that at low temperatures, which is attributed to a lower sensitivity of the confinement energy to size fluctua-
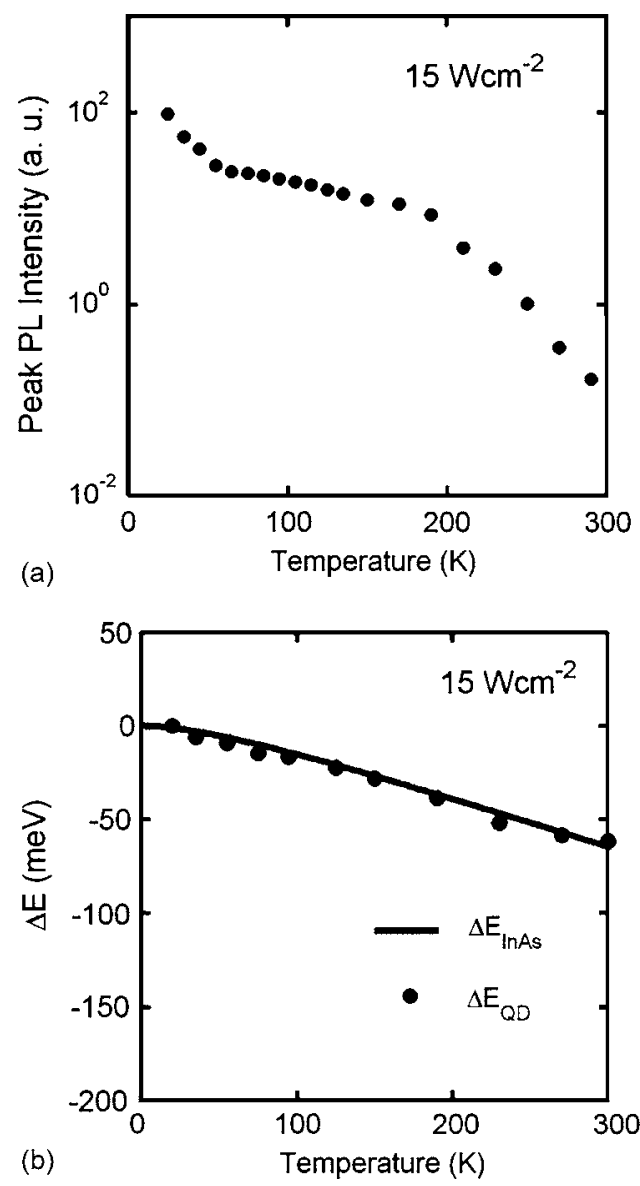

FIG. 6. (a) Variation of photoluminescence peak intensity with temperature for sample C; (b) shift of PL peak energy with respect to peak energy at $20 \mathrm{~K}$ of the active quantum dots of sample $\mathrm{C}$ (solid circles) together with change of bulk InAs band gap (line) with temperature.

tion for larger quantum dots. Thus the second feature that is noteworthy in our data is that we observe a small and continuous increase in the linewidth as the temperature is increased. These features indicate that there is little or no carrier redistribution or transfer (including transfer to or from the dots in the seed layer) with increasing temperature and the size distribution amongst the dots in the active dot layer is very small. The increase in linewidth observed in the range of $20-300 \mathrm{~K}$ reflects thermal (phonon) broadening. We further studied the contribution of carrier-carrier scattering to the linewidth broadening. As shown in Fig. 5(c), with the increase of excitation intensity from 0.05 to $60 \mathrm{~W} / \mathrm{cm}^{2}$ at room temperature, the linewidth only shows a small increase, from 17.5 to $18.4 \mathrm{meV}$. This suggests that the homogeneous broadening of our QDs is primarily due to phonon scattering.

In order to verify the absence, or minimal occurrence, of carrier redistribution amongst the active dots with increasing temperature due to size variations, we have further examined the temperature-dependent PL data. Figure 6(a) shows the temperature-dependent PL peak intensity for sample C. The trend of the data is similar to that of Fig. 5(a) for the integrated PL intensity. A peaking in the intensity in the temperature range of $100-200 \mathrm{~K}$, which is usually observed for InAs/GaAs quantum dots, ${ }^{27}$ and signifies carrier redistribution into larger dots, is again absent. Figure 6(b) shows the 
plot of $\Delta E_{\mathrm{QD}}$ with temperature, where $\Delta E_{\mathrm{QD}}$ is the shift of the PL peak energy with temperature measured with respect to the peak energy at $20 \mathrm{~K}$. The solid line along with the data is the variation of the band gap of InAs with temperature, calculated using the Varshni equation. ${ }^{29}$ The agreement is excellent, which signifies that carrier redistribution due to size variation in dots is minimal, or absent. If there is a carrier transfer to larger dots due to size variations, a redshift of the luminescence peak larger than the decrease in the InAs band gap will be observed. ${ }^{27}$ The difference between $\Delta E_{\text {InAs }}$ and $\Delta E_{\mathrm{QD}}$ is similar to a Stokes shift, the difference between the PL peak and the absorption maximum. Therefore, there is overwhelming evidence that carrier redistribution in the bilayer dot system is negligible and this stems from the size uniformity of the dots in the active layer, also reflected by the narrow PL linewidths.

A fair question to ask is how the measured ensemble PL linewidths compare with those from single dots. It is known that at low temperatures $(\leqslant 20 \mathrm{~K})$, linewidths much smaller than $1 \mathrm{meV}$ have been measured in the PL emission from single $\operatorname{In}(\mathrm{Ga})$ As/GaAs quantum dots. ${ }^{30-33}$ Therefore, we conclude that our measured linewidth of $10.6 \mathrm{meV}$ at $20 \mathrm{~K}$ in sample $\mathrm{C}$ is determined predominantly by inhomogeneous broadening, which includes heterostructure interface roughness. At $300 \mathrm{~K}$, single-dot PL linewidths in the range of $12-17 \mathrm{meV}$, for interlevel spacing in the range of $30-45 \mathrm{meV}$, have been measured. ${ }^{30}$ These linewidths are in agreement with the calculated homogeneous broadening due to LA- and LO-phonon scattering. In our dots, the energy difference between the ground and first excited electron states, determined by theory and experiment, is $\sim 60 \mathrm{meV}$. ${ }^{34}$ We have made microphotoluminescence measurements on sample $\mathrm{C}$ wherein the emission from five to seven quantum dots was probed through a $250-\mathrm{nm}$ aperture in a gold film deposited on the sample. Linewidths of $13-15 \mathrm{meV}$ are measured. Therefore, it is reasonable to assume that the measured linewidth of $17.5 \mathrm{meV}$ at $300 \mathrm{~K}$ in sample $\mathrm{C}$ is primarily limited by homogeneous broadening.

\section{CONCLUSION}

In conclusion, we have optimized the molecular-beam epitaxial growth of InAs/GaAs quantum dot bilayers, in which a layer of stressor dots is followed by a GaAs barrier layer and the layer of active dots. By tuning the growth parameters, we have obtained photoluminescence linewidths of $10.6 \mathrm{meV} \quad\left(\lambda_{\text {peak }}=1.305 \mu \mathrm{m}\right) \quad$ and $\quad 17.5 \mathrm{meV} \quad\left(\lambda_{\text {peak }}\right.$ $=1.4 \mu \mathrm{m})$. The analysis of the temperature-dependent photoluminescence data confirms that there is a very high degree of size uniformity amongst the dots in the active layer and there is no evidence of carrier redistribution with an increase of temperature. On comparison with the PL data for single quantum dots reported in the literature, it is evident that the linewidth of $17.5 \mathrm{meV}$ measured at $300 \mathrm{~K}$ is limited by homogeneous broadening.

\section{ACKNOWLEDGMENTS}

The help and useful discussions with Dr. S. Chakrabarti are gratefully acknowledged. The authors also wish to thank J. Topolancik and S. Chakravarty for the micro-PL measurements. This work was supported by the Center for Optoelectronic Nanostructured Semiconductor Technologies, a DARPA UPR award, under Contract No. HR0011-04-10040.

${ }^{1}$ K. Kamath, P. Bhattacharya, T. Sosnowski, T. Norris, and J. Phillips, Electron. Lett. 32, 1374 (1996).

${ }^{2}$ D. L. Huffaker, G. Park, Z. Zou, O. B. Shchekin, and D. G. Deppe, Appl. Phys. Lett. 73, 2564 (1998).

${ }^{3}$ A. R. Kovsh et al., Electron. Lett. 35, 1161 (1999).

${ }^{4}$ M. Sugawara, N. Hatori, T. Akiyama, Y. Nakata, and H. Ishikawa, Jpn. J. Appl. Phys., Part 2 40, 488 (2001).

${ }^{5}$ P. Borri et al., Appl. Phys. Lett. 79, 2633 (2001).

${ }^{6}$ Z. Bakonyi, H. Su, G. Onishchukov, L. F. Lester, A. L. Gray, T. C. Newell, and A. Tunnermann, IEEE J. Quantum Electron. 39, 1409 (2003).

${ }^{7}$ K. W. Berryman, S. A. Lyon, and M. Segev, Appl. Phys. Lett. 70, 1861 (1997).

${ }^{8}$ J. Philips, P. Bhattacharya, S. W. Kennerly, D. W. Beekman, and M. Dutta, IEEE J. Quantum Electron. 35, 936 (1999).

${ }^{9}$ D. Pan, E. Towe, and S. Kennerly, Appl. Phys. Lett. 73, 1937 (1998).

${ }^{10}$ S. Sangu, K. Kobayashi, A. Shojiguchi, and M. Ohtsu, Phys. Rev. B 69, 115334 (2004)

${ }^{11}$ A. Kiraz, M. Atature, and A. Imamoglu, Phys. Rev. A 69, 032305 (2004).

${ }^{12}$ J. Vuckovic and Y. Yamamoto, Appl. Phys. Lett. 82, 2374 (2003).

${ }^{13}$ J. Vuckovic, D. Fattal, C. Santori, and G. S. Solomon, Appl. Phys. Lett. 82, 3596 (2003).

${ }^{14}$ P. Michler, A. Kiraz, C. Becher, W. V. Schoenfeld, P. M. Petroff, L. D. Zhang, E. Hu, and A. Imamoglu, Science 290, 2282 (2000).

${ }^{15}$ H. Lee, W. D. Yang, and P. C. Sercel, Phys. Rev. B 55, 9757 (1997).

${ }^{16}$ V. M. Ustinov et al., Appl. Phys. Lett. 74, 2815 (1999).

${ }^{17}$ I. Mukhametzhanov, R. Heitz, J. Zeng, P. Chen, and A. Madhukar, Appl. Phys. Lett. 73, 1841 (1998).

${ }^{18}$ S. Krishna, J. Sabarinathan, K. Linder, and P. Bhattacharya, J. Vac. Sci. Technol. B 18, 1502 (2000).

${ }^{19}$ E. C. Le Ru, P. Howe, T. S. Jones, and R. Murray, Phys. Rev. B 67, 165303 (2003).

${ }^{20}$ T. Yang, J. Tatebayashi, S. Tsukamoto, M. Nishioka, and Y. Arakawa, Appl. Phys. Lett. 84, 2817 (2004).

${ }^{21}$ K. Nishi, H. Saito, S. Sugou, and J.-S. Lee, Appl. Phys. Lett. 74, 1111 (1999).

${ }^{22}$ Q. Xie, J. L. Brown, R. L. Jones, J. E. Van Nostrand, and K. D. Leedy, Appl. Phys. Lett. 76, 3082 (2000).

${ }^{23}$ M. H. Baier, S. Watanabe, E. Pelucchi, and E. Kapon, Appl. Phys. Lett. 84, 1943 (2004).

${ }^{24}$ Q. Xie, A. Madhukar, P. Chen, and N. P. Kobayashi, Phys. Rev. Lett. 75, 2542 (1995).

${ }^{25}$ G. S. Solomon, J. A. Trezza, A. F. Marshall, and J. S. Harris, Phys. Rev. Lett. 76, 952 (1996).

${ }^{26}$ A. Endoh, Y. Nakata, Y. Sugyama, M. Takatsu, and N. Yokoyama, Jpn. J. Appl. Phys., Part 1 38, 1085 (1999).

${ }^{27}$ A. Polimeni, A. Patané, M. Henini, L. Eaves, and P. C. Main, Phys. Rev. B 59, 5064 (1999).

${ }^{28}$ S. Sanguinetti, M. Henini, M. Grassi Alessi, M. Capizzi, P. Frigeri, and S. Franchi, Phys. Rev. B 60, 8276 (1999).

${ }^{29}$ Y. P. Varshni, Physica (Amsterdam) 34, 149 (1967).

${ }^{30}$ M. Grundmann et al., Phys. Rev. Lett. 74, 4043 (1995).

${ }^{31}$ K. Matsuda, K. Ikeda, T. Saiki, H. Tsuchiya, H. Saito, and K. Nishi, Phys. Rev. B 63, 121304 (2001).

${ }^{32}$ M. Bayer and A. Forchel, Phys. Rev. B 65, 041308 (2002).

${ }^{33}$ E. Dekel, D. Gershoni, E. Ehrenfreund, D. Spektor, J. M. Garcia, and P. M. Petroff, Phys. Rev. Lett. 80, 4991 (1998).

${ }^{34}$ A. A. Dikshit and J. M. Pikal, IEEE J. Quantum Electron. 40, 105 (2004). 